\title{
Modifications of the Method for Calculating Absolute Drug Bioavailability
}

Wei Jiang, Juan-juan Yang, Lei Cao, Xue Xiao, Xiao-lian Shi, Yong-xiao Cao

Department of Pharmacology, Xi'an Jiaotong University College of Medicine

Received, March 13, 2016; Revised, May 18, 2016, Accepted, May 19, 2016, Published, May 23 $3^{\text {rd }} 2016$.

\begin{abstract}
Purpose: Absolute bioavailability (F) is calculated as the ratio of the area under the plasma drug concentration-time curve (AUC) between extravascular administration and intravenous injection. However, as distribution of a drug after intravenous administration does not reach an equilibrium in the body during the distribution phase, the plasma drug concentration at this phase does not reflect the total amount of drug in the body. The goal of this paper was to analyze the insufficiencies of the method for calculating on absolute bioavailability and to propose a modification to improve the calculation. Methods: Literature reporting absolute bioavailability published during 1983-2014 was searched for ten drug candidates. Plasma drug concentrations representing the amount of drug in the body were then calculated at each time point during the distribution phase according to the plasma drug concentration-time relationship during the elimination phase. Results: The AUC values based on the distribution equilibrium drug concentrations following intravenous injection were $75 \% \pm 11 \%$ of the actually measured drug concentrations in the literature. The absolute bioavailability values in the literature were $76 \% \pm 12 \%$ of the actual bioavailability based on the AUCs from distribution-equilibrium drug concentrations. Conclusions: The present method underestimates the absolute drug bioavailability and should be modified to represent the data more accurately.
\end{abstract}

This article is open to POST-PUBLICATION REVIEW. Registered readers (see "For Readers") may comment by clicking on ABSTRACT on the issue's contents page.

\section{INTRODUCTION}

As an important indicator in pharmacokinetics, absolute bioavailability is defined as the fraction of an administered drug that reaches the circulation system $(F)$, which is the ratio between the amount of drug presented in the systemic circulation and the total administered dose. To calculate $\mathrm{F}$, the area under the curve of the plasma drug concentration (AUC) after intravenous (iv) and extravascular (ev) administration is obtained [1-4]. The AUC following iv administration, however, reflects the amount of a drug in the blood circulation before distribution into the rest of the body. On the other hand, following ev administration, the drug will distribute while it is being absorbed so that, for the majority of drugs, the distribution process is masked by the absorption phase. Hence, as a marker of the amount of the drug in the body, the plasma drug concentration during the distribution phase is an over-estimation. This investigation aimed to analyze these insufficiencies and to suggest possible modifications for determining absolute drug bioavailability.
The concentrations of a drug in different organs or tissues are different. Nonetheless, when the drug distribution reaches pseudo-equilibrium, i.e., equal concentration in the permeable tissues, the amount of a drug in an organ or a tissue is equal the drug concentration multiply by the volume of the organ or the tissue. The apparent volume of distribution, $\mathrm{V}_{\mathrm{d}}$, is defined as the theoretical volume of body fluid that is required to dissolve the drug at the same concentration as that in plasma. In linear pharmacokinetics, $V_{d}$ of a drug is constant regardless of the administration routes. Hence, the total amount of a drug (A) in the body equals $\mathrm{V}_{\mathrm{d}}$ multiple by its blood drug concentration (C) at equilibrium:

$$
\mathrm{A}=\mathrm{V}_{\mathrm{d}} \cdot \mathrm{C}
$$

Corresponding Author: Yong-xiao Cao, $\mathrm{PhD}$, Professor, Department of Pharmacology, Xi'an Jiaotong University College of Medicine, 76 Yanta West Road, Xi'an, 710061, P.R. China, E-mail: yxy@mail.xjtu.edu.cn 
The fraction of an administered drug that reaches the circulatory system $(\mathrm{F})$ is the ratio between the amount of drug being bioavailable and the total amount of drug administered. When a drug is injected via a vein, F equals 1 . Therefore,

$$
\mathrm{F}=\frac{\mathrm{Vd} \cdot \mathrm{C} e v}{\mathrm{Vd} \cdot \mathrm{C} i v}=\frac{\mathrm{C} e v}{\mathrm{C} i v}=\frac{\mathrm{AUC} e v}{\mathrm{AUCiv}}
$$

Where, $\mathrm{C}_{e v}$ and $\mathrm{C}_{i v}$ are the plasma drug concentrations of $e v$ and $i v$ administration of the same dose, respectively. While during the pre-pseudo-equilibrium time, the amount in the reachable sites of the body is different between $i v$ and $e v$ administration, the concentration values are assumed to be markers of the drug in the body. This, however, is not true until the post-pseudoequilibrium has reached.

Taking the two-compartment model for example, after $i v$ administration, the drug reaches the circulatory system instantaneously. The plasma drug concentration-time curve shows a distribution phase and an elimination phase. First, the drug emerges in the central compartment, such as the vascular system, and those organs or tissues that take the drug in as fast as it is distributed in plasma. The distribution into the organs and/or tissues (deep tissue compartment) that are slow in letting the drug permeate occurs is significantly slower pace (Fig. 1). Graphically, this process is presented by two distinct phases, distribution $(\alpha)$ and elimination ( $\beta$ ) (Fig. 2).

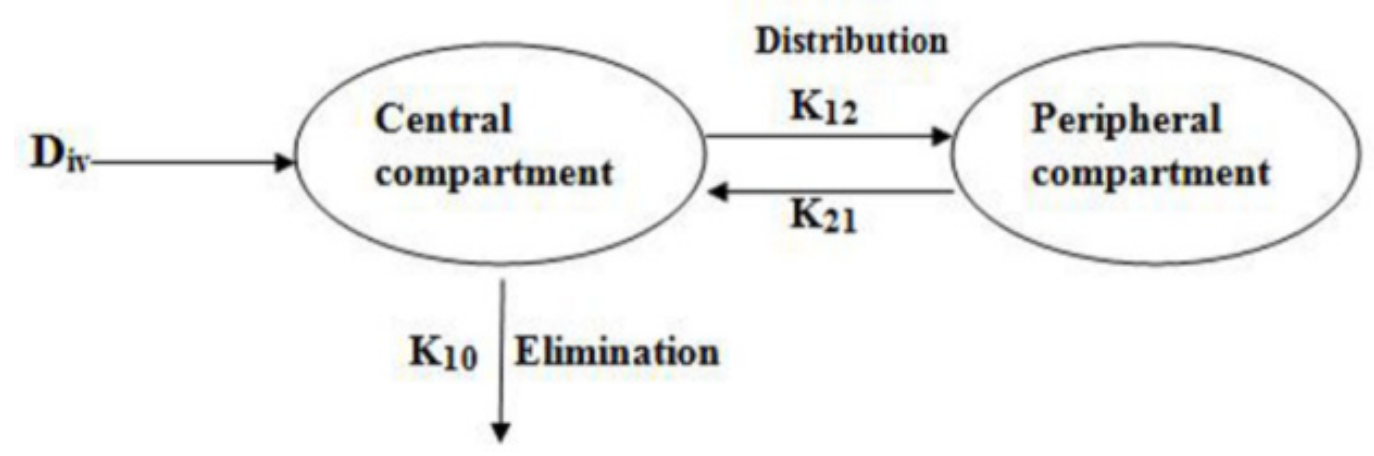

Figure.1. Two-compartment model for $i v$ administration of a drug.

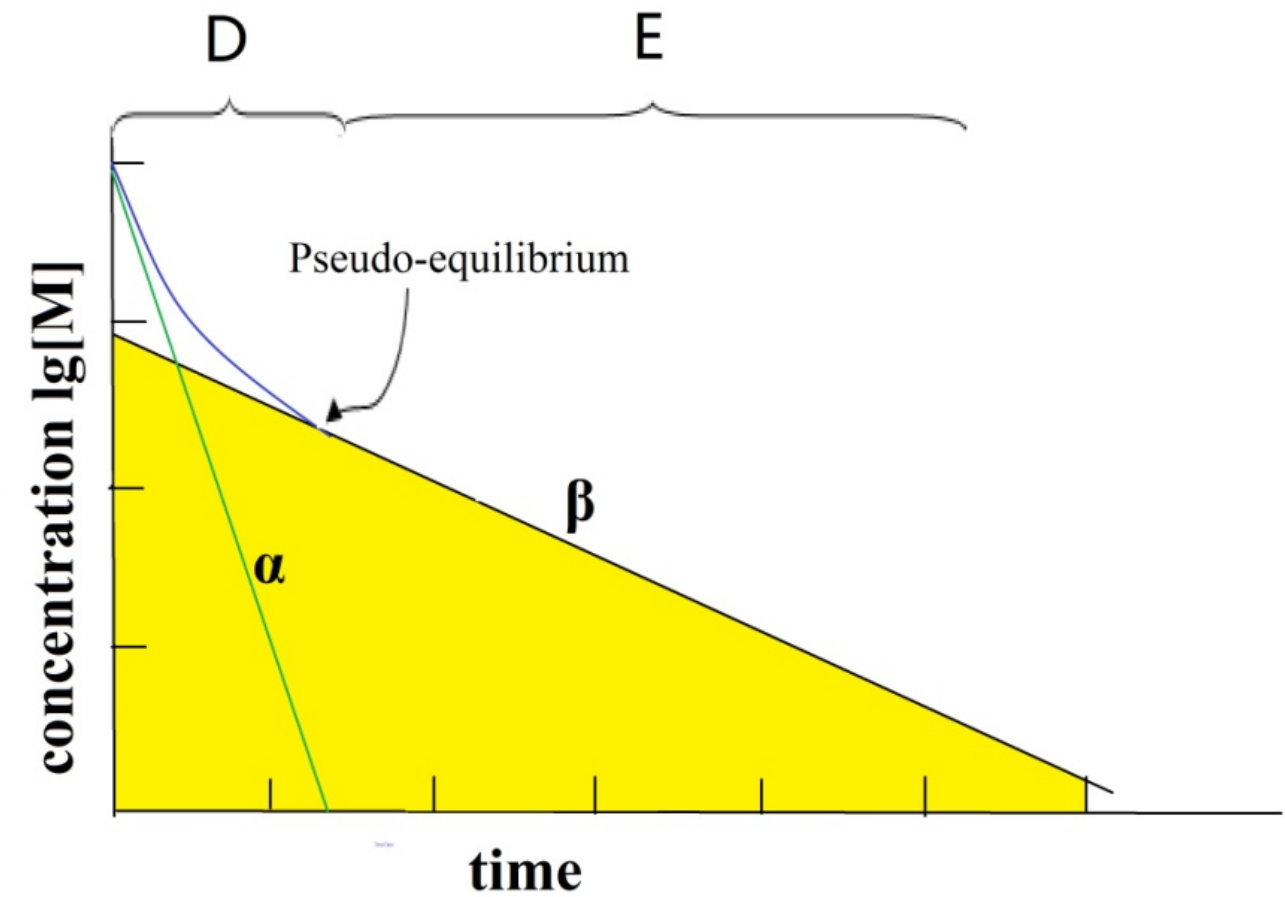

Figure.2. The plasma concentration-time curve of a drug via $i v$ administration. D, distribution phase; E, elimination phase. 
In contrast to $i v$ route, with $e v$ administration, a drug molecule gradually enters the central compartment to be distributed to other organs and/or tissues. In such cases, the pseudo-equilibrium point may not be seen if the absorption is slower than distribution process.

Absolute drug bioavailability is typically calculated using actually measured values of plasma drug concentrations, hence, to calculate AUC, the contribution of the effect of route of administration on the distribution kinetics is one often ignored.

From equation 3 below, which describes a log-linear process where $\log C$ is the drug concentration, $t$ is the time, $\beta$ is the elimination rate constant, $\log \mathrm{B}$ is they-intercept of the $\log$ linear elimination line and $\mathrm{B}$ is the maximum plasma drug concentration when the distribution has reached pseudo-equilibrium.

$$
\begin{aligned}
& \log \mathrm{C}=-\frac{\beta}{2.303} \mathrm{t}+\log \mathrm{B} \\
& \mathrm{C}=\mathrm{B} \cdot e^{-\beta \mathrm{t}}
\end{aligned}
$$

AUC can be calculated from:

$$
\mathrm{AUC}=\int_{0}^{+\infty} \mathrm{C}=\int_{0}^{+\infty} \mathrm{B} \cdot e^{-\beta \mathrm{t}}=\frac{\mathrm{B}}{\beta}
$$

Or $B / \beta$ reflects the AUC of the dose after attainment of pseudo-equilibrium. For a drug with a two or multi-compatment model, this value is obviously smaller than AUC calculated using the actually measured drug concentrations that includes those during distribution phase. For example, we used the data of Muck et al.[5] to calculate the true $\mathrm{F}$ that was adjusted for the distribution process. Healthy young male volunteers received either a single $100 \mu \mathrm{g} i v$ dose or $200 \mu \mathrm{g}$ oral tablets of cerivastatin to determine the absolute bioavailability. The plasma cerivastatin concentrations after iv administration were determined using the Microsoft Paint software according to the concentration-time curve (Table 1). The data for the plasma cerivastatin concentrations and times were then input into the DAS 3.1.5 software (Shanghai, China). The results showed that the plasma concentration-time curve fits to the two-compartment model. The $\mathrm{AUC}_{\infty}, \mathrm{B}$, and $\beta$ were $7.89 \mu \mathrm{g} \cdot \mathrm{h} / \mathrm{L}, 1.804$ and 0.349 , respectively. This $\mathrm{AUC}_{\infty}$ is 1.02 times that reported in the literature
[5], suggesting that the recovered data are very closed to the actual data. The values of $B$ and $\beta$ are entered into equation 5, and the AUC based on equilibrium is calculated as the follows:

$$
\mathrm{AUC}=\frac{1.804}{0.349}=5.17
$$

The result is $5.17 \mu \mathrm{g} \cdot \mathrm{h} / \mathrm{L}$. In the literature, the oral AUC is $9.34 \mu \mathrm{g} \cdot \mathrm{h} / \mathrm{L}$. Adjusting for the dose, the $i v$ and oral AUCs are input into equation 4 to determine the absolute bioavailability $\mathrm{F}$, which is 0.903 .

In addition, the AUC based on equilibrium can be calculated using another method. According to equation 3 , after $\log \mathrm{C}$ and $t$ of every points in the elimination phase are linearly regressed, the $\beta / 2.303(-0.152)$ and $\log B(0.256)$ can be obtained. Therefore, the equation representing the plasma cerivastatin concentration-time relationship at the elimination phase is as follows:

$$
\log C=-0.152 t+0.256
$$

By inputting $t$ values from every time point into the linear equation 7 , the corresponding drug concentrations is calculated (Table 1). We did so using DAS 3.1.5 to calculate the AUC following iv administration. The result was $5.22 \mu \mathrm{g} \cdot \mathrm{h} / \mathrm{L}$. In the literature, the oral AUC was $9.34 \mu \mathrm{g} \cdot \mathrm{h} / \mathrm{L}$ for twice as much a dose. Hence, an $F$ of $89.5 \%$ was obtained.

\section{METHODS}

A search of the literature from 1983 to 2014 was undertaken using PubMed (https://www.ncbi.nlm.nih.gov/pubmed) using the keywords "absolute bioavailability". We downloaded 171 papers, and only two of which included data regarding the plasma drug concentrations after $i v$ administration. Thirty-two papers showed legible figures of plasma drug concentration-time curves. Microsoft Paint software, which has a digital scale, was used to convert the plasma drug concentrations from the concentration-time curves to numerical values for each time point. The recovered numerical values of plasma drug concentrations were then input into the DAS 3.1.5 software, and the information about the compartment model and the pharmacokinetic parameters, including $\mathrm{AUC}, \mathrm{B}$, and $\beta$, were 
calculated. In this case, we used only the ratio of the AUC calculated from the recovered plasma drug concentrations and the actual measurements was larger than 0.95 and smaller than 1.05 , indicating that the recovered plasma drug concentrations were close to the actual measured ones for further study. In this study, we only analyzed the deviation of $F$ based on the two-compartment model. As a result, 10 data sets, 2 of which were original data from published literature [6] and 8 of which were transformed from the plasma concentration-time graphs [5,7-12], met the preconditions and were further analyzed.
Information on the types of administration, doses, AUCs drugs after $e v$ administration as well as absolute bioavailability were recorded for 10 selected studies (Table 2). They all were described as having the two-compartment model, and used the actually measured drug concentrations. Regarding experimental subjects, one study used broiler chickens [11], one used Sprague-Dawley rats [12], and the rest used human subjects. All of the studies used oral administration as the ev administration method, except for one that used inhalation [7]. The information from the 10 studies are depicted in Table 2.

Table 1. The mean plasma concentrations of cerivastatin.

\begin{tabular}{ccc}
\hline Time, $\mathrm{h}$ & Measured concentrations, $\mu \mathrm{g} / \mathrm{L}$ & Adjusted concentrations, $\mu \mathrm{g} / \mathrm{L}$ \\
\hline 0.083 & 13.01 & 1.75 \\
0.167 & 5.61 & 1.70 \\
0.250 & 3.33 & 1.65 \\
0.333 & 2.29 & 1.61 \\
0.500 & 1.81 & 1.52 \\
0.750 & 1.49 & 1.39 \\
1.000 & 1.31 & 1.27 \\
1.500 & 1.06 & 1.07 \\
2.000 & 0.99 & 0.90 \\
2.500 & 0.83 & 0.75 \\
3.000 & 0.56 & 0.63 \\
4.000 & 0.36 & 0.45 \\
6.000 & 0.20 & 0.22 \\
8.000 & 0.18 & 0.11 \\
\hline
\end{tabular}

Table 2. Information from the examined studies.

\begin{tabular}{|c|c|c|c|c|c|c|}
\hline \multirow[t]{2}{*}{ No } & \multirow[t]{2}{*}{ Drugs } & \multicolumn{2}{|c|}{ Doses (mg) } & \multicolumn{2}{|c|}{$\operatorname{AUC}(\mathrm{ng} \cdot \mathrm{h} / \mathrm{ml})$} & \multirow[t]{2}{*}{$\mathrm{F}$} \\
\hline & & $i v$ & po & $i v$ & po & \\
\hline 1 & Cefatrizine & 528 & 505 & 37800 & 27000 & 75.0 \\
\hline 2 & Cefatrizine & 1037 & 1010 & 82900 & 37000 & 46.8 \\
\hline 3 & triamcinolone ${ }^{*}$ & 0.4 & 0.8 & 10.3 & 5.1 & 25.0 \\
\hline 4 & Melatonin & 2.0 & 4.0 & 27.2 & 8.4 & 15.9 \\
\hline 5 & Nifedipine & 2.5 & 10 & 78 & 191 & 63.0 \\
\hline 6 & Nifedipine & 2.5 & 10 & 89 & 301 & 86.0 \\
\hline 7 & Almotriptan & 3.0 & 25 & 78 & 443 & 69.1 \\
\hline 8 & Cerivastatin & 0.1 & 0.2 & 7770 & 9340 & 60.0 \\
\hline 9 & Deoxynivalenol $^{* *}$ & $0.75^{\# \#}$ & 0.75 & 103.3 & 21.4 & 19.3 \\
\hline 10 & diosbulbin $\mathrm{B}^{\#}$ & $0.5^{\# \#}$ & 32 & 1644.8 & 1987.3 & 2.0 \\
\hline
\end{tabular}


Microsoft Paint software was used to convert the plasma drug concentration-time curves from the papers into numerical values for each time point. The recovered plasma drug concentrations were used to calculate the AUC, and only those values from the recovered plasma drug concentrations that were equal to the AUC values in the papers were considered for further analysis. The distribution-equilibrium plasma drug concentrations were calculated using equation 3 , and then applied to calculate the AUC of the drugs after $i v$ administration. Equation 5 can be also used to calculate AUCs based on the distribution equilibrium $\left(\mathrm{AUC}_{\mathrm{b}}\right)$.

\section{RESUTS AND DISCUSSION}

Data presented in Table 3 demonstrate that the $\mathrm{AUC}_{\mathrm{b}}$ values were lower than those based on actual measurements $\left(\mathrm{AUC}_{\mathrm{a}}\right)$. The $\mathrm{AUC}_{\mathrm{b}} / \mathrm{AUC}_{\mathrm{a}}$ ratio was $0.75 \pm 0.11$. The absolute bioavailability based on the distribution balance $\left(\mathrm{F}_{\mathrm{b}}\right)$ was calculated using equation 2 , and the results showed that $F_{b}$ was higher than the absolute bioavailability based on the actually measured concentrations $\left(F_{a}\right)$. The values of $\mathrm{F}_{\mathrm{a}} / \mathrm{F}_{\mathrm{b}}$ for the 10 data sets ranged from 0.462 to 0.880 . The mean and standard deviation was $76 \% \pm 12 \%$, which suggests that the $F$ value of drugs is underestimated using the present method.

A drug administered via an $i v$ route has an absolute bioavailability of 1.0 , whereas the absolute bioavailability of drugs administered by other routes is less than one unless it is fully bioavailable. AUC reflects the amount of a drug in the blood, and $\mathrm{AUC}_{e v} / \mathrm{AUC}_{i v}$ presents the absolute bioavailability following $e v$ administration. The assumption herein, is that in both $i v$ and $e v$ doses have reached equilibrium in the body. This assumption is reached for both routes of administration when the drug follows the conventional one compartment model right after administration, which happens. After iv administration of multi-compartment model drugs, on the other hand, the circulating drug concentration measured reflect the amount in the body only when pseudo-equilibrium has reached.

Table 3. AUC and absolute bioavailability based on the distribution-balanced drug concentrations.

\begin{tabular}{llllllll}
\hline No & $\begin{array}{l}\mathrm{AUC}_{\mathrm{r}} \\
(\mathrm{ng} \cdot \mathrm{h} / \mathrm{ml})\end{array}$ & $\frac{\mathrm{AUC}_{\mathrm{r}}}{\mathrm{AUC}_{\mathrm{a}}}$ & $\begin{array}{l}\mathrm{AUC} \\
(\mathrm{ng} \cdot \mathrm{h} / \mathrm{ml})\end{array}$ & $\frac{\mathrm{AUC}_{\mathrm{b}}}{\mathrm{AUC}_{\mathrm{a}}}$ & $\mathrm{F}_{\mathrm{b}}$ & $\frac{\mathrm{F}_{\mathrm{a}}}{\mathrm{F}_{\mathrm{b}}}$ & Reference \\
\hline 1 & 37800 & 1.000 & 29104 & 0.770 & 95.7 & 0.784 & {$[6]$} \\
2 & 82900 & 1.000 & 71433 & 0.862 & 53.2 & 0.880 & {$[6]$} \\
3 & 10.288 & 0.999 & 8.287 & 0.805 & 30.9 & 0.809 & {$[7]$} \\
4 & 26.54 & 0.975 & 20.58 & 0.757 & 20.4 & 0.779 & {$[8]$} \\
5 & 81.093 & 1.040 & 64.149 & 0.822 & 74.4 & 0.846 & {$[9]$} \\
6 & 89.096 & 1.001 & 69.424 & 0.780 & 108.4 & 0.793 & {$[9]$} \\
7 & 81.292 & 1.040 & 54.109 & 0.693 & 98.4 & 0.702 & {$[10]$} \\
8 & 7890 & 1.015 & 5169 & 0.655 & 90.3 & 0.655 & {$[5]$} \\
9 & 107.29 & 1.039 & 51.15 & 0.495 & 41.7 & 0.462 & {$[11]$} \\
10 & 1642 & 0.999 & 1288 & 0.861 & 2.4 & 0.830 & {$[12]$} \\
Mean & & 1.01 & & 0.75 & & 0.76 & \\
SD & & 0.02 & & 0.11 & & 0.12 &
\end{tabular}

$\mathrm{AUC}_{\mathrm{r}}$ : The AUC is calculated from recovered plasma drug concentration.

$\mathrm{AUC}_{\mathrm{b}}$ : The AUC is calculated from distribution balanced plasma concentration.

$\mathrm{AUC}_{\mathrm{a}}$ : The AUC is calculated from on actual measure plasma concentration.

$\mathrm{F}_{\mathrm{b}}$ : The absolute bioavailability is based on the distribution-balanced drug concentrations.

$\mathrm{F}_{\mathrm{a}}$ : The absolute bioavailability is based on actual measurements of drug concentrations. 
Hence, the AUC following $i v$ doses of drugs with multi-compartment is an over-estimation of that that reflects the amount that reached the permeable pars of the entire body. These results in underestimation of absolute bioavailability measured based on $\mathrm{AUC}_{e v} / \mathrm{AUC}_{i v}$.

To further calculate corresponding AUCs, plasma drug concentrations in the distribution equilibrium are calculated based on the formula for the plasma drug concentration-time relationship in the elimination phase. In addition, $\mathrm{B} / \beta$ is a simpler method for calculating the AUC. Although some differences in the results of the two methods exist, these differences are very small and negligible. Our results showed that the AUC values based on drug concentrations at the distribution equilibrium is 0.75 times those of the AUCs in the corresponding published papers. The average $F_{a}$ calculated from the $\mathrm{AUC}_{\mathrm{iv}}$ values based on the actually measured concentrations is $76 \%$ of the $\mathrm{F}_{\mathrm{b}}$ from the $\mathrm{AUC}_{\mathrm{iv}}$ values based on the distribution equilibrium, indicating that the $\mathrm{F}$ was underestimated by the present method, and corrections should be performed.

Drug distribution model can impact the application of the method. For a drug with a one-compartment model, the drug distribution reaches equilibrium immediately. After intravascular administration, there is an ignorable or no distribution phase. Therefore, the result of this extrapolated method is the same or similar one of the current method. So, the method is suitable for drugs with a two-compartment and multi-compartment, but not for one-compartment model. However, the number of drugs with one-compartment model is less.

\section{CONCLUSION}

The distribution of a drug after intravenous administration does not reach an equilibrium in the body during the distribution phase, and the AUC values based on the actual measurements of drug concentrations does not reflect the total amount of drug in the body. The absolute bioavailability values based on the AUCs from actual measurements of drug concentrations are lower than the actual bioavailability based on the AUCs from distribution-equilibrium drug concentrations. The present method underestimates the absolute drug bioavailability and should be modified.

\section{ACKNOWLEDGMENT}

This research is supported by a grant from the $\mathrm{Xi}$ 'an Jiaotong University teaching reform research project (27).

\section{Author contributions}

Conceived and designed the experiments: YXC and LC. Performed the experiments: WJ and JJY. Analyzed the data: WJ and XX. Contributed analytical tools: XLS. Wrote the paper: WJ, LC, and YXC.

\section{REFERENCES}

1. Rolan P, Lim S, Sunderland V, Liu Y, Molnar V (2014) The absolute bioavailability of racemic ketamine from a novel sublingual formulation. $\mathrm{Br} \mathrm{J}$ Clin Pharmacol 77: 1011-1016. DOI: 10.1111/bcp. 12264.

2. Devreese M, Broekaert N, De Mil T, Fraeyman S, De Backer P, et al. (2014) Pilot toxicokinetic study and absolute oral bioavailability of the Fusarium mycotoxin enniatin B1 in pigs. Food Chem Toxicol 63: 161-165. DOI: 10.1016/j.fct.2013.11.005.

3. Kramer WG, Reuning RH (1978) Use of area under the curve to estimate absolute bioavailability of digoxin. J Pharm Sci 67: 141-142.DOI:10.1002/jps. 2600670145.

4. Guirguis MS, Saeed S, Jamali F.(2001). "Pharmacokinetics of celecoxib in the presence and absence of interferon-induced acute inflammation in the rat: application of a novel HPLC assay." J Pharm Pharm Sci 4(1): 1-6.

5. Muck W, Ritter W, Ochmann K, Unger S, Ahr G, et al. (1997) Absolute and relative bioavailability of the HMG-CoA reductase inhibitor cerivastatin. Int $\mathrm{J}$ Clin Pharmacol Ther 35: 255-260.

6. Pfeffer M, Gaver RC, Ximenez J (1983) Human intravenous pharmacokinetics and absolute oral bioavailability of cefatrizine. Antimicrob Agents Chemother 24: 915-920. DOI:10.1128/AAC.24.6. 915.

7. Argenti D, Shah B, Heald D (1999) A pharmacokinetic study to evaluate the absolute bioavailability of triamcinolone acetonide following inhalation administration. J Clin Pharmacol 39: 695-702. DOI:10.1177/00912709922008335.

8. DeMuro RL, Nafziger AN, Blask DE, Menhinick AM, Bertino JS, Jr. (2000) The absolute bioavailability of oral melatonin. J Clin Pharmacol 40: 781-784. DOI:10.1177/00912700022009422.

9. Rashid TJ, Martin U, Clarke H, Waller DG, Renwick AG, et al. (1995) Factors affecting the absolute bioavailability of nifedipine. $\mathrm{Br} \mathrm{J}$ Clin 
Pharmacol 40: 51-58. DOI: 10.1111/j.1365-2125 1995.tb04534.x.

10. Jansat JM, Costa J, Salva P, Fernandez FJ, Martinez-Tobed A (2002) Absolute bioavailability, pharmacokinetics, and urinary excretion of the novel antimigraine agent almotriptan in healthy male volunteers. J Clin Pharmacol 42: 1303-1310. DOI: 10.1177/0091270002042012006.

11. Osselaere A, Devreese M, Goossens J, Vandenbroucke V, De Baere S, et al. (2013) Toxicokinetic study and absolute oral bioavailability of deoxynivalenol, T-2 toxin and zearalenone in broiler chickens. Food Chem Toxicol 51: 350-355. DOI:10.1016/j.fct.2012.10.006.

12. Yang B, Wang X, Liu W, Zhang Q, Chen K, et al. (2013) Gender-related pharmacokinetics and absolute bioavailability of diosbulbin B in rats determined by ultra-performance liquid chromatography-tandem mass spectrometry. J Ethnopharmacol 149: 810-815. DOI:10.1016/j.jep. 2013.08.010. 